\title{
Genetic testing for cerebral cavernous malformations
}

\author{
Yeltay Rakhmanov ${ }^{1}$, Paolo Enrico Maltese ${ }^{1 \star}$, Carla Marinelli ${ }^{1}$, Leonardo D’Agruma ${ }^{2}$, Tommaso Beccari ${ }^{3}$, \\ Munis Dundar ${ }^{4}$ and Matteo Bertelli ${ }^{1,5}$
}

\begin{abstract}
Cavernous cerebral malformations (CCM) are vascular malformations of the brain and spinal cord. CCM affect up to $0.5 \%$ of the general population, predisposing to headaches, seizures, cerebral hemorrhage and focal neurological deficit. CCM may be familial or sporadic. Familial forms have autosomal dominant inheritance. This Utility Gene Test was prepared on the basis of an analysis of the literature and existing diagnostic protocols. It is useful for confirming diagnosis, as well as for differential diagnosis, couple risk assessment and access to clinical trials.
\end{abstract}

Keywords: Cavernous cerebral malformations, CCM, EBTNA UTILITY GENE TEST

'MAGI's Lab, Rovereto, Italy

${ }^{2}$ Medical Genetic Unit, IRCCS - Hospital

"Casa Sollievo della Sofferenza" San

Giovanni Rotondo - Italy

${ }^{3}$ Department of Pharmaceutical Sciences, University of Perugia, Perugia, Italy

${ }^{4}$ Department of Medical Genetics, Erciyes University Medical School, Kayseri, Turkey

${ }^{5}$ MAGI Euregio, Bolzano, Italy

*Corresponding author: P. E. Maltese E-mail: paolo.maltese@assomagi.org

DOI: $10.2478 /$ ebtj-2018-0046
(C) 2018 Authors. This work was licensed under the Creative Commons AttributionNonCommercial-NoDerivs 3.0 License.

\section{Cerebral cavernous malformations}

(Other synonyms: Familial cavernous angioma, cavernous angiomatous malformations, hyperkeratotic cutaneous capillary-venous malformations associated with cerebral capillary malformations)

\section{General information about the disease}

Cerebral cavernous malformations (CCM, OMIM disease: 116860) are a specific type of vascular malformation of the central nervous system. Their morphological structure consists of well-circumscribed collections of dilated, thin-walled vascular channels lined by simple endothelium and thin fibrous adventitia, containing blood that is usually clotted or in a state of decomposition (1).

The overall prevalence of all CCM has been estimated at 1/200 to 1/1,000 individuals. Familial CCM accounts for about $20 \%$ of all CCM cases with an estimated prevalence of $1 / 5,000-1 / 10,000$ and is therefore rare, unlike the common sporadic CCM. A strong founder effect has been detected in Hispanic-American families affected by CCM (2).

There are sporadic and familial forms of CCM. Sporadic cases are characterized by a lack of family history of the disease and usually MRI evidence of a single lesion $(3,4)$, although multiple lesions have been reported $(5,6)$. In contrast, familial cases mostly exhibit multiple lesions that progress in number and size over time (7).

Diagnostic procedures should be based on identification of symptoms (seizure disorder, focal neurological deficits, nonspecific headaches, cerebral haemorrhage, vascular skin lesions, capillary malformations, hyperkeratotic cutaneous capillary venous malformations, venous malformations, red macules, and/or nodular venous malformations, retinal cavernomas and rare choroidal hemangiomas) with the aid of high-resolution MR imaging using either gradient echo (GRE) or susceptibility-weighting (SWI) and angiography (8), and collecting information on family history.

Differential diagnosis should consider arteriovenous malformations, venous malformations, telangiectases, vascular tumors such as hemangioblastomas (including 
those seen in Von Hippel-Lindau syndrome) and vascular malformations associated with Sturge-Weber syndrome.

CCM has autosomal dominant inheritance with incomplete penetrance and include:

- CCM1 (OMIM disease 116860) - KRIT1 (OMIM gene 604214);

- CCM2 (OMIM disease 603284) - CCM2 (OMIM gene 607929);

- CCM3 (OMIM disease 603285) - PDCD10 (OMIM gene 609118).

Somatic mutations in the three genes have been identified in CCM lesions from both sporadic and familial patients (9). Gault et al. found somatic mutations in the KRIT1 gene in vascular endothelial cells lining cavernomas (10).

Pathogenic variants may include missense, nonsense, splicing, small insertions, small deletions, small indels, gross insertions and gross deletions.

\section{Aims of the test}

- To determine the gene defect responsible for the disease;

- To confirm clinical diagnosis;

- To assess the recurrence risk and perform genetic counselling for at-risk/affected individuals.

\section{Test characteristics}

\section{Specialist centers/ Published Guidelines}

The test is listed in the Orphanet database and is offered by 9 accredited medical genetic laboratories in the EU, and in the GTR database, offered by 12 accredited medical genetic laboratories in the US.

Guidelines for clinical use of the test are described in Genetics Home Reference (ghr.nlm.nih.gov) and Gene Reviews (7).

\section{Test strategy}

A multi-gene next generation sequencing panel is used for the detection of nucleotide variations in coding exons and flanking introns of the above genes.

Potentially causative variants and regions with low coverage are Sanger-sequenced. Sanger sequencing is also used for family segregation studies.

Multiplex Ligation Probe Amplification (MLPA) is used to detect insertions and deletions in KRIT1, CCM2 and PDCD10.

To perform molecular diagnosis, a single sample of biological material is normally sufficient. This may be $1 \mathrm{ml}$ peripheral blood in a sterile tube with $0.5 \mathrm{ml} \mathrm{K}$ EDTA or $1 \mathrm{ml}$ saliva in a sterile tube with $0.5 \mathrm{ml}$ ethanol $95 \%$. Sampling rarely has to be repeated.

Gene-disease associations and the interpretation of genetic variants are rapidly developing fields. It is therefore possible that the genes mentioned in this note may change as new scientific data is acquired. It is also possible that genetic variants today defined as of "unknown or uncertain significance" may acquire clinical importance.

\section{Genetic test results}

\section{Positive}

Identification of pathogenic variants in the above genes confirms the clinical diagnosis and is an indication for family studies.

A pathogenic variant is known to be causative for a given genetic disorder based on previous reports, or predicted to be causative based on loss of protein function or expected significant damage to proteins or protein/protein interactions. In this way it is possible to obtain a molecular diagnosis in new/other subjects, establish the risk of recurrence in family members and plan preventive and/or therapeutic measures.

\section{Inconclusive}

Detection of a variant of unknown or uncertain significance (VUS): a new variation without any evident pathogenic significance or a known variation with insufficient evidence (or with conflicting evidence) to indicate it is likely benign or likely pathogenic for a given genetic disorder. In these cases, it is advisable to extend testing to the patient's relatives to assess variant segregation and clarify its contribution. In some cases, it could be necessary to perform further examinations/tests or to do a clinical reassessment of pathological signs.

\section{Negative}

The absence of variations in the genomic regions investigated does not exclude a clinical diagnosis but suggests the possibility of:

- alterations that cannot be identified by sequencing, such as large rearrangements that cause loss (deletion) or gain (duplication) of extended gene fragments;

- sequence variations in gene regions not investigated by this test, such as regulatory regions (5' and 3' UTR) and deep intronic regions;

- variations in other genes not investigated by the present test.

\section{Unexpected}

Unexpected results may emerge from the test, for example information regarding consanguinity, absence of family correlation or other genetically-based diseases.

\section{Risk for progeny}

In autosomal dominant transmission, the probability that an affected carrier transmit the variant to his/her children is $50 \%$ in any pregnancy, irrespective of the sex of the child conceived.

\section{Limits of the test}

The test is limited by current scientific knowledge regarding the gene and disease.

Analytical sensitivity (proportion of positive tests when the genotype is truly present) and specificity (proportion of negative tests when the genotype is not present)

NGS Analytical sensitivity $>99.99 \%$, with a minimum coverage of 10X; Analytical specificity $99.99 \%$. 
SANGER Analytical sensitivity $>99.99 \%$; Analytical specificity 99.99\%.

MLPA Analytical sensitivity $>99.99 \%$; Analytical specificity 99.99\%.

Clinical sensitivity (proportion of positive tests if the disease is present) and clinical specificity (proportion of negative tests if the disease is not present)

Clinical sensitivity: the variations in the aforementioned genes are associated with CCM, but in many cases, these are individual variations (identified in one or few families) and total epidemiological data is therefore not available.

Clinical specificity: Data not available.

\section{Prescription appropriateness}

The genetic test is appropriate when:

a) the patient meets the diagnostic criteria for CCM;

b) the sensitivity of the test is greater than or equal to that of tests described in the literature.

\section{Clinical utility}

\begin{tabular}{|l|c|}
\hline Clinical management & Utility \\
\hline Confirmation of clinical diagnosis & Yes \\
\hline Differential diagnosis & Yes \\
\hline Couple risk assessment & Yes \\
\hline $\begin{array}{l}\text { Availability of clinical trials can be checked on-line at } \\
\text { https://clinicaltrials.gov/ }\end{array}$
\end{tabular}

\section{References}

1. Porter PJ, Willinsky RA, Harper W, Wallace MC. Cerebral cavernous malformations: natural history and prognosis after clinical deterioration with or without hemorrhage. J Neurosurg 1997; 87(2): 190-97.

2. Tournier-Lasserve E. Familial cerebral cavernous malformation. Orphanet encyclopedia. 2014

3. Labauge P, Laberge S, Brunereau L, Levy C, Tournier-Lasserve E. Hereditary cerebral cavernous angiomas: clinical and genetic features in 57 French families. Societe Francaise de Neurochirurgie. Lancet 1998; 352: 1892-97.

4. Petersen TA, Morrison LA, Schrader RM, Hart BL. Familial versus sporadic cavernous malformations: differences in developmental venous anomaly association and lesion phenotype. AJNR Am J Neuroradiol 2010; 31(2): 377-82.

5. Verlaan DJ, Laurent SB, Sure U, Bertalanffy H, Andermann E, Andermann F, Rouleau G.A, Siegel A.M. CCM1 mutation screen of sporadic cases with cerebral cavernous malformations. Neurology 2004; 62(7): 1213-15.

6. Labauge $P$, Brunereau L, Coubes $P$, Clanet $M$, Tannier C, Laberge $\mathrm{S}$, Levy $\mathrm{C}$. Appearance of new lesions in two nonfamilial cerebral cavernoma patients. Eur Neurol 2001; 45(2): 83-88.

7. Rigamonti D, Hadley MN, Drayer BP, Johnson PC, Hoenig-Rigamonti K, Knight JT, Spetzler RF. Cerebral cavernous malformations. Incidence and familial occurrence. N Engl J Med 1988; 319(6): 343-47.

8. Morrison L, Akers A. Cerebral Cavernous Malformation, Familial. 2003 Feb 24. In: Adam MP, Ardinger HH, Pagon RA, et al., editors. GeneReviews $^{\circledR}$ 1993-2018. Available from: https://www.ncbi.nlm. nih.gov/books/NBK1293/

9. Choquet H, Pawlikowska L, Lawton MT, Kim H. Genetics of cerebral cavernous malformations: current status and future prospects. J Neurosurg Sci 2015; 59(3): 211-20.

10. Gault J, Awad IA, Recksiek P, Shenkar R, Breeze R, Handler M, Kleinschmidt-DeMasters BK. Cerebral cavernous malformations: somatic mutations in vascular endothelial cells. Neurosurgery 2009; 65(1): 138-44. 